\title{
Efecto antioxidante y citoprotector del tocosh de Solanum tuberosum 'papa' en la mucosa gástrica de animales de experimentación
}

\author{
Antioxidant and cytoprotector effects of Solanum tuberosum 'papa' tocosh in gastric
} mucose in experimental animals

\author{
Miguel Hernán Sandoval Vegas ${ }^{1}$, Janeth Tenorio Mucha², Aldo Tinco Jayo², \\ Rudi A. Loli Ponce ${ }^{1}$, Segundo Calderón Pinillos ${ }^{3}$ \\ 'Centro de Investigación de Bioquímica y Nutrición. Facultad de Medicina, Universidad Nacional Mayor de San Marcos, Lima, Perú. \\ ${ }^{2}$ Escuela de Formación Profesional, Farmacia y Bioquímica, Facultad de Ciencias Biológicas, Uinversidad Nacional San Cristóbal de \\ Humanga, Ayacucho, Perú. \\ ${ }^{3}$ Departamento Académico de Nutrición, Facultad de Medicina. UNMSM.
}

\begin{abstract}
Resumen
El tocosh es un producto alimenticio obtenido por una técnica de conservación andina y que tiene propiedades nutritivas y terapéuticas. Objetivo: Demostrar la capacidad antioxidante y el efecto citoprotector del tocosh de Solanum tuberosum 'papa' en la mucosa gástrica de animales de experimentación. Diseño: Experimental. Institución: Centro de Investigación de Bioquímica y Nutrición, Facultad de Medicina, Universidad Nacional Mayor de San Marcos, Lima, Perú. Material biológico: Tocosh seco y molido administrado a ratas albinas. Intervenciones: A 6 grupos de ratas albinas machos $(200 \pm 50 \mathrm{~g})$ se les dividió en: (GI) solución $\mathrm{NaCl}$ 0,9\%, $10 \mathrm{~mL} / \mathrm{kg}$; (GII) etanol al 70\% a $10 \mathrm{~mL} / \mathrm{kg}$; (GIII, IV y V) Tocosh equivalente a $900 \mathrm{mg} / \mathrm{kg}, 1800 \mathrm{mg} / \mathrm{kg}$ y $2700 \mathrm{mg} / \mathrm{kg}$, respectivamente, y (GVI) sucralfato $30 \mathrm{mg} / \mathrm{kg}$. En todos los casos, una hora después se indujo injuria con etanol $70^{\circ}$ a $10 \mathrm{~mL} / \mathrm{kg}$ y por laparotomía abdominal se obtuvo el tejido gástrico. Principales medidas de resultados: Porcentaje de citoprotección gástrica en imagen digitalizada por image analysis software for plant disease quantification y capacidad antioxidante por lipoperoxidación método espectrofotométrico de la reacción de especies reactivas al ácido tiobarbitúrico (TBARS). Resultados: La marcha fitoquímica identificó compuestos fenólicos, alcaloides, triterpenoides y esteroides, azúcares reductores y aminoácidos libres como metabolitos secundarios. Las dosis de 2700 $\mathrm{mg} / \mathrm{kg}$ y $900 \mathrm{mg} / \mathrm{kg}$ resultaron en 0,72 y $1,81 \mathrm{nmol} / \mathrm{g}$ tejido de lipoperoxidación, respectivamente. La dosis de $1800 \mathrm{mg} / \mathrm{kg}$ protegió un $97 \%$ del área de la mucosa gástrica, $2700 \mathrm{mg} / \mathrm{kg}$ un $95 \%$ y la de $900 \mathrm{mg} / \mathrm{kg}, 88 \%(\mathrm{p}<0,05)$. La dosis de $1800 \mathrm{mg} / \mathrm{kg}$ exhibió mejor efecto citoprotector y la de $2700 \mathrm{mg} / \mathrm{kg}$ mejor actividad antioxidante, comparada con sucralfato $30 \mathrm{mg} / \mathrm{kg}$. Conclusiones: El tocosh de Solanum tuberosum 'papa' tuvo efecto citoprotector y actividad antioxidante.
\end{abstract}

Palabras clave: Tocosh, mucoprotección, mucosa gástrica, Solamun tuberosum.

\section{Abstract}

Tocosh is a nutritious product obtained by an Andean preservation technique with nutritive and therapeutic properties. Objective: To demonstrate antioxidant and cytoprotector effects of Solanum tuberosum 'papa' tocosh in the gastric mucosa of experimental animals. Design: Experimental. Institution: Biochemistry and Nutrition Research Center, Faculty of Medicine, Universidad Nacional Mayor de San Marcos, Lima, Peru. Biologic material: Dry and grounded administered to albino rats. Interventions: Six groups of male albino rats $(200 \pm 50 \mathrm{~g}$ ) were divided in: (GI) NaCl 0,9\% solution, $10 \mathrm{~mL} / \mathrm{kg}$; (GII) ethanol $70 \% 10 \mathrm{~mL} / \mathrm{kg}$; (GIII, IV y V) Tocosh equivalent to 900 $\mathrm{mg} / \mathrm{kg}, 1800 \mathrm{mg} / \mathrm{kg}$, and $2700 \mathrm{mg} / \mathrm{kg}$ respectively, and (GVI) sucralfate $30 \mathrm{mg} / \mathrm{kg}$. One hour after, in all cases injury with ethanol $70^{\circ}$ $10 \mathrm{~mL} / \mathrm{kg}$ was induced and gastric tissue was obtained by abdominal laparotomy. Main outcome measures: Percentage of gastric cytoprotection in digitalized image by image analysis software for plant disease quantification, and antioxidant ability by lipoperoxidation spectrophotometric method of reactive species reaction to thiobarbituric acid (TBARS). Results: The phytochemist progress identified phenolic compounds, alcaloids, triterpenoids and steroids, reducing sugars and free aminoacids as secondary metabolites. The 2700 $\mathrm{mg} / \mathrm{kg}$ and $900 \mathrm{mg} / \mathrm{kg}$ doses resulted respectively in 0.72 and $1.81 \mathrm{nmol} / \mathrm{g}$ lipoperoxidation tissue. The $1800 \mathrm{mg} / \mathrm{kg}$ doses protected $97 \%$ of the gastric mucosa, $2700 \mathrm{mg} / \mathrm{kg} \mathrm{95 \%} \mathrm{and} \mathrm{the} 900 \mathrm{mg} / \mathrm{kg}, 88 \%(\mathrm{p}<0.05)$. The $1800 \mathrm{mg} / \mathrm{kg}$ doses exhibited better cytoprotector effect and the $2700 \mathrm{mg} / \mathrm{kg}$ doses better antioxidant activity, compared with sucralfate $30 \mathrm{mg} / \mathrm{kg}$. Conclusions: Solanum tuberosum 'papa' tocosh showed cytoprotector effect and antioxidant activity.

Keywords: Tocosh, mucoprotection, gastric mucosa, Solamun tuberosum.

An Fac med. 2015;76(1):15-20 / doi:10.15381/anales.v76i1.11070 


\section{INTRODUCCIÓN}

El Perú posee cerca de $72 \%$ de las zonas de vida en el mundo, hecho que da como resultado una variedad intensa de especies en flora y fauna, que desde tiempos antiguos estuvieron a disposición de los pobladores, influyendo notablemente en el desarrollo de sus hábitos alimenticios y costumbres ${ }^{(1)}$. Situaciones provocadas por factores ambientales o sociopolíticos forzaron a los pobladores a observar, descubrir y aplicar técnicas particulares a los alimentos con el fin de obtener mejores cosechas, conservarlos y prolongar su vida útil. El tocosh es una técnica tradicional andina de conservación in situ ${ }^{(2)}$. La palabra tocosh deriva del vocablo togosh, palabra quechua que significa arrugado y fermentado ${ }^{(3)}$, y es el resultado de la fermentación bacteriana de productos andinos como la papa, el maíz, olluco o la aracacha, almacenada en pozos construidos en la tierra, envueltos en paja o 'ichu' y presionados mecánicamente con piedras bajo una corriente de agua proveniente de un manantial ${ }^{(4,5)}$. Este tratamiento confiere a los productos propiedades nutritivas y terapéuticas aprovechadas solo por quienes las conocen y las consumen y que podría ser una alternativa natural para paliar, prevenir o curar algunas enfermedades ${ }^{(6)}$.

Desde épocas incaicas, los pobladores de las regiones de Ancash, Huánuco y Junín utilizan tocosh como medicamento, siendo la mazamorra de tocosh o tocosh api la forma de consumo más conocida. Se caracteriza por su olor desagradable, que es lo primero en percibirse, peculiaridad que no limita su consumo o comercialización, afirmándose por conocimiento empírico que contiene penicilina natural y que entre sus innumerables beneficios es capaz de proteger la mucosa gástrica de daño o inflamación ${ }^{(3,4)}$, debiéndose verificar de manera científica esta propiedad atribuida.

De acuerdo a costumbres populares, este producto es usado en el posparto, resfrío, neumonía, en la curación de heridas, como antibacteriano, cicatrizante de hemorroides y de úlcera gás- trica, para evitar las infecciones gastrointestinales y mal agudo de altura o 'soroche'. Además, podría ser un antibiótico, energizante, probiótico eficaz y de muy bajo costo ${ }^{(7)}$.

Es importante mencionar que la gastritis es una enfermedad inflamatoria aguda o crónica de la mucosa gástrica producida por factores exógenos y endógenos. Entidad de elevada morbilidad a nivel mundial, en el Perú es una de las causas que con más frecuencia motivan la consulta gastroenterológica ${ }^{(8)}$. En la actualidad, esta enfermedad ha aumentado debido al estrés, la bacteria Helicobacter pylori y el elevado consumo de café y alcohol. La alteración histológica más evidente en la mucosa gástrica, originada por la gastritis, es la respuesta inflamatoria. En los procesos inflamatorios participan diferentes tipos de células que, al ser atraídas al sitio de la lesión, liberan gran variedad de mediadores químicos, como citoquinas, eicosanoides y radicales libres, los cuales amplifican la respuesta inflamatoria y aumentan la proliferación celular, con la posibilidad, según la intensidad y persistencia, de inducir a errores de replicación celular y facilitar el desarrollo del cáncer gástrico ${ }^{(9,10)}$.

El presente trabajo de investigación pretende evaluar la capacidad antioxidante y el efecto citoprotector del tocosh de Solanum tuberosum 'papa' en la mucosa gástrica, en un modelo experimental en ratas albinas.

\section{MÉTODOS}

El estudio tuvo diseño básico-experimental. Se utilizó tocosh de Solanum tuberosum 'papa' seca, adquirido en el mercado Raez Patiño del Distrito de Huancayo, Provincia de Huancayo, región Junín. Fue triturado hasta obtener un polvo fino, se retiraron las impurezas, se pesó según las dosis y luego fue reconstituido en agua caliente $\left(90^{\circ} \mathrm{C}\right)$ y sometido a decocción, hasta obtener un gel. Finalmente, se dejó enfriar a temperatura ambiente. Para la identificación cualitativa de los componentes del tocosh, se realizó la marcha fitoquímica a la decocción, siguiendo la metodología propuesta por Lock de Ugaz ${ }^{(11)}$.

El experimento se realizó en 60 ratas sprague dawley machos adultos adquiridos del bioterio de la Universidad $\mathrm{Na}$ cional Agraria La Molina (UNALM), de $200 \pm 25 \mathrm{~g}$ de peso, criados y alimentados en las mismas condiciones. Los animales fueron divididos aleatoriamente en 6 grupos: (G1) control negativo: solución de cloruro de sodio $0,9 \%$ a $10 \mathrm{~mL} / \mathrm{kg}$ de peso; (G2) control positivo: etanol al $70 \%$ a $10 \mathrm{~mL} / \mathrm{kg}$ de peso; (G3) tocosh de Solanum tuberosum, a $900 \mathrm{mg} / \mathrm{kg}$ de peso del animal + etanol al $70 \%$ a $10 \mathrm{~mL} / \mathrm{kg}$ de peso; (G4) tocosh a $1800 \mathrm{mg} / \mathrm{kg}$ de peso + etanol al $70 \%$ a $10 \mathrm{~mL} / \mathrm{kg}$ de peso; (G5) tocosh a $2700 \mathrm{mg} / \mathrm{kg}$ de peso + etanol al $70 \%$ a $10 \mathrm{~mL} / \mathrm{kg}$ del animal; y (G6) sucralfato a $30 \mathrm{mg} / \mathrm{Kg}+$ etanol al $70 \%$ a $10 \mathrm{~mL} / \mathrm{kg}$. En todos los casos la vía de administración fue por canulación orogástrica, en estado de ayuno sólido de 24 horas; los animales fueron mantenidos con solución de glucosa al 5\% hasta 4 horas antes de la canulación. La administración del etanol al 70\% se realizó una hora después de cada tratamiento con tocosh o sucralfato, para inducir gastritis y úlceras gástricas. Después de 60 minutos, los animales fueron anestesiados con éter vaporizado y se les practicó laparotomía y gastrectomía. El estómago de cada animal fue extendido y fijado en superficie adherente, para luego ser fotografiados, digitilizados y trasferidos a una computadora, para su análisis con el software ASSESS Image Analysis Software for Plant Disease Quantification, en el cual se identificó y cuantificó las áreas: área total, ulcerada y no ulcerada de la mucosa del estómago en $\mathrm{cm}^{2}$.

Se expresó los resultados como daño de la mucosa gástrica en porcentaje de área ulcerada $\left(\mathrm{cm}^{2}\right)$, de acuerdo al cálculo siguiente:

Área ulcerada $\left(\mathrm{cm}^{2}\right)=$ Área total de la mucosa gástrica $\left(\mathrm{cm}^{2}\right)$ - área no ulcerada $\left(\mathrm{cm}^{2}\right)$

Daño $=\%$ área ulcerada $=$ (área ulcerada) $/($ área total de la mucosa gástrica) $\times 100$ 
Para observar la capacidad antioxidante se midió el grado de estrés oxidativo producido por el etanol $70 \%$, para lo cual se pesó aproximadamente $0,5 \mathrm{~g}$ de tejido gástrico para realizar la lipoperoxidación mediante la prueba de TBARS (determinación de las especies reactivas al ácido tiobarbitúrico). Se midió en espectrofotómetro a una longitud de onda de $535 \mathrm{~nm}$.

Se expresó los resultados de acuerdo al siguiente cálculo:

Lipoperoxidación $=\mathrm{Abs} /(\varepsilon x \mathrm{l}) \times 1 / 10^{-9} \times(5 \mathrm{~L}) /$

$1000 \times 1 /$ (w tejido (g) )

Donde: Abs es la absorbancia, I la longitud de la celda de $1 \mathrm{~cm}, \varepsilon=$ coeficiente de extinción molar $=1,56 \times 10^{5}(\mathrm{~mol} / \mathrm{L})^{-1} \mathrm{~cm}^{-1}$ y w peso del tejido.

Los datos obtenidos fueron sometidos al análisis de varianza (ANOVA), prueba de Tukey para comparar la diferencia entre grupos y prueba de Dunnet para la comparación con respecto a grupos control a un nivel de confianza del $95 \%$ (p 0,05).

\section{RESULTADOS}

La decocción de tocosh de papa (mazamorra) presentó como características alta viscosidad y propiedades mucilaginosas: Entre sus componentes se encontraron compuestos fenólicos, alcaloides, triterpenos, esteroides, azúcares reductores y aminoácidos libres, como se puede apreciar en la tabla 1.

El grado de lipoperoxidación fue significativamente menor en el grupo que consumió la más alta dosis de tocosh (G5), es decir con mayor efecto antioxidante, y fue significativamente mayor en el grupo al que se le administró solo etanol (G2) p <0,01, es decir, donde se observó mayor estrés oxidativo. El tocosh mostró capacidad antioxidante en todas las dosis en comparación al grupo con etanol, que tuvo alto valor de lipoperoxidación $(\mathrm{p}<0,055)$ (ver figura 1$)$.

El área de lesión sobre la mucosa gástrica causada por el alcohol fue de

Tabla 1. Marcha fitoquímica de la decocción de tocosh de Solanum tuberosum "papa" seco.

\begin{tabular}{ccc} 
Reactivo & Metabolito & Resultado \\
$\mathrm{FeCl}_{3}$ & Compuestos fenólicos & +++ \\
Shinoda $\left(\mathrm{Mg}^{0}+\mathrm{HCl}\right)$ & Flavonoides & - \\
Dragendorff & Alcaloides & +++ \\
Mayer & Alcaloides & +++ \\
Wagner & Alcaloides & +++ \\
Lieberman & Triterpenoides y esteroides & ++ \\
Baljet & Lactonas & - \\
Borntrager & Quinonas & - \\
Kedde & Glicósidos cardiotónicos & - \\
Benedict & Azúcares reductores & ++ \\
Ninhidrida & Aminoácidos libres & +++ \\
$\mathrm{Na}_{2} \mathrm{CO}_{3}+$ Luz UV & Catequinas & - \\
\hline
\end{tabular}

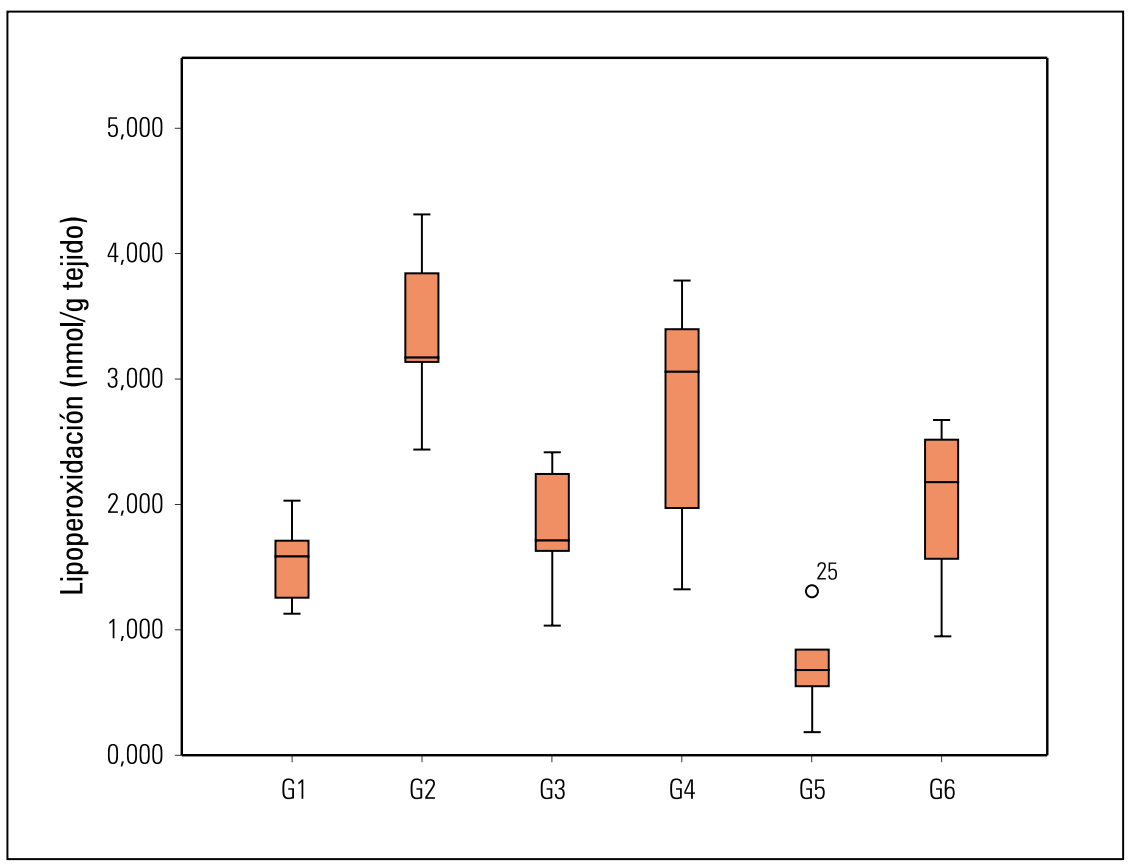

Figura 1. Efecto antioxidante por lipoperoxidación de mucosa gástrica del tocosh de Solanum tuberosum 'papa'.

27,4\%. Áreas de menor lesión se observó en los grupos a los que se les administró el tocosh con dosis de 1800 y $2700 \mathrm{mg} / \mathrm{kg}$ de peso (daño $2,9 \%$ y $4,5 \%$, respectivamente), en ambos casos con porcentaje de área protegida mayor que el grupo con sucralfato (ver tabla 2).

\section{DISCUSIÓN}

En el estudio fitoquímico de la decocción de tocosh de Solanum tuberosum 'papa' seco, se identificó cualitativamente como metabolitos secundarios compuestos fenólicos, alcaloides, triterpenoides y esteroides, azúcares re- 
Tabla 2. Área gástrica y porcentaje de citoprotección - daño de la mucosa.

\begin{tabular}{ccccc} 
Grupo & Tratamiento & Área total $\mathrm{cm}^{2}$ & \% Protección & \% Daño \\
G1 & NaCl 0,9\% 10 mL/kg & $10,3 \pm 1,2$ & 99,3 & 0,7 \\
G2 & Etanol $70 \% 10 \mathrm{~mL} / \mathrm{kg}$ & $10,7 \pm 1,3$ & 72,6 & 27,4 \\
G3 & Tocosh $900 \mathrm{mg} / \mathrm{kg}+$ etanol & $9,2 \pm 0,6$ & 88,1 & 11,9 \\
G4 & Tocosh $1800 \mathrm{mg} / \mathrm{kg}+$ etanol & $9,3 \pm 1,3$ & 97,1 & $2,9^{*}$ \\
G5 & Tocosh $2700 \mathrm{mg} / \mathrm{kg}+$ etanol & $10,9 \pm 0,8$ & 95,5 & $4,5^{*}$ \\
G6 & Sucralfato $30 \mathrm{mg} / \mathrm{kg}+$ etanol & $10,03 \pm 1,01$ & 92,7 & $7,3^{*}$ \\
\hline
\end{tabular}

(*) $p<0,05$ respecto a $G 2$

ductores y aminoácidos libres (tabla 1). Investigaciones referidas a la misma especie, S. tuberosum, indican que esta contiene: compuestos fenólicos, predominantemente ácido clorogénico; glicoalcaloides, siendo los principales la chaconina y la solanina; flavonoides; azúcares reductores (glucosa y fructosa) y antocianinas. Resaltan que el mayor componente del material seco es el almidón, amilosa y amilopectina del almidón, así como sus demás componentes dependen de la variedad y el cultivo. La presencia de estos le confiere la característica mucilaginosa que posee $^{(12)}$.

Para el caso del tocosh, Naupari y col. (4) identificaron esteroides y triterpenos, alcaloides, compuestos fenólicos, flavonoides, cumarinas y taninos. Por su parte, Gutiérrez ${ }^{(13)}$ señala que los componentes químicos mayoritarios del tocosh son de estructura y naturaleza polar y detectó la presencia de azúcares reductores, aminoácidos, alcaloides y esteroides. En ese sentido, podemos inferir que el material crudo de tocosh S. tuberosum (el polvo seco) no difiere en su composición fitoquímica con el tocosh preparado por decocción (a modo de mazamorra). Se ha encontrado diferencias en la composición químico-bromatológico entre la papa y el tocosh ${ }^{(4)}$, como consecuencia de los fenómenos de germinación y fermentación que pueden asegurar cambios en sus componentes, mejorar su valor nutritivo ${ }^{(5)}$ e incluso remover glicoalcaloides tóxicos ${ }^{(12)}$.

La evaluación de la actividad antioxidante se realizó por la determinación de las especies reactivas al ácido tiobarbitúrico (TBARS), midiendo la actividad inhibidora de la formación de radicales libres por la producción de malondialdehído como indicador de lipoperoxidación; y para causar estrés oxidativo se usó el etanol, ya que se relaciona positivamente con los niveles de TBARS ${ }^{(14)}$; los valores de la prueba TBARS son un reflejo de la lipoperoxidación por agresión del alcohol ${ }^{(15,16)}$ (MDA-TBA).

Como se ha observado en los resultados (figura 1), en todos los casos el tocosh mostró actividad antioxidante y con mayor diferencia estadística en la dosis de $2700 \mathrm{mg} / \mathrm{kg}$ de peso $(\mathrm{p}<0,01)$. Paralelamente, la lipoperoxidación fue mayor en el grupo en que se administró etanol, que en los grupos a los que, además del alcohol, se les administró previamente el tocosh, lo que demuestra su actividad antioxidante. Hemos encontrado que la actividad antioxidante del tocosh no es dependiente de la dosis (ver figura 1) y podemos inferir que se debe a sus componentes. $\mathrm{Al}$ realizar el análisis comparativo entre los tratamientos con tocosh con las dosis de $900 \mathrm{mg} / \mathrm{kg}, 1800 \mathrm{mg} / \mathrm{kg}$ y $2700 \mathrm{mg} /$ $\mathrm{kg}$, y sucralfato $30 \mathrm{mg} / \mathrm{kg}$, se observa que existen diferencias en su capacidad de producción del complejo MDATBA $(p<0,05)$. La prueba de Dunnet determina que la dosis de $2700 \mathrm{mg} / \mathrm{kg}$ de tocosh de Solanum tuberosum 'papa' tiene mejor actividad antioxidante que el sucralfato, $0,72 \pm 0,41 \mathrm{nmol} / \mathrm{g}$ tejido frente a $1,98 \pm 0,72 \mathrm{nmol} / \mathrm{g}$ tejido de lipoperoxidación $(\mathrm{p}<0,05)$.

La alta capacidad antioxidante de la papa se debe al aporte de sus componentes. La papa contiene carotenoides, principalmente xantofilas, entre ellas, luteína, zexantina y violaxantina; compuestos fenólicos, en su mayoría ácido clorogénico; flavonoides, predominantemente catequina y epicatequina. Además, se ha informado la presencia de vitamina $C^{(17,18)}$. Coetzer ${ }^{(19)}$ refiere que los polifenoles presentes en $S$. tuberosum tienen capacidad antioxidante como agentes quelantes de iones de metales de transición, uniéndose a estos y reduciendo su capacidad de generar radicales libres; además, tienen la capacidad de inhibir, activar o proteger enzimas específicas que inhiben oxigenasas celulares.

Entre los lípidos que contiene la papa se encuentra el $\alpha$-tocoferol ${ }^{(20)}$, y el tocosh de papa brinda un gran aporte probiótico, por la presencia de lactobacilos al final del proceso de fermentación de la papa ${ }^{(6)}$; es decir, el tocosh de papa ofrece una mezcla natural de probióticos y $\alpha$-tocoferol y según señalan Senol y col. ${ }^{(21)}$, esta mezcla de probióticos y $\alpha$-tocoferol reduce la lipoperoxidación en la mucosa gástrica inducida por etanol.

Por estudios previos ${ }^{(22)}$, hemos observado menores niveles de lipoperoxidación en la mucosa gástrica tratada con sobrenadante de $5 \mathrm{~mL} / \mathrm{kg}$ del zumo de S. tuberosum, la que tuvo un valor de $5,22 \pm 3,19 \mathrm{nmol} / \mathrm{g}$ tejido; en cambio, los valores de lipoperoxidación del tocosh muestran mejor actividad antioxidante, hecho que podría ser explicado por Rosenthal y col. ${ }^{(23)}$, que examinaron el nivel antioxidante en especies frescas y almacenadas y determinaron que los tubérculos almacenados tienen mayor actividad antioxidante que los tubérculos frescos y que este incremento se debió a la temporada de frío; y, para el caso del tocosh, este se produce con una técnica de conservación en frío ${ }^{(1,6)}$; de allí las propiedades observadas en nuestra investigación.

También hemos correlacionado la actividad citoprotectora de Croton palanostigma 'sangre de grado' con la formación de una capa de protección física a la mucosa y la presencia de grupos 
sulfhidrilos en la mucosa, evidenciando menor lipoperoxidación con respecto al alcohol 96\% (24). Podríamos afirmar que para el tocosh de papa se cumple el mismo principio para la citoprotección de la mucosa gástrica. Sin embargo, el rol de los radicales libres inducidos por la lipoperoxidación en la generación de injuria crónica en la mucosa gástrica es desconocido ${ }^{(16)}$.

La actividad citoprotectora se evidencia tras la injuria por etanol $70 \%$ a la mucosa gástrica en todos los grupos. El análisis de varianza (ANOVA) determinó que existen diferencias significativas $(\mathrm{p}<0,05)$ entre los tratamientos administrados (tabla 2). La prueba de Tukey mostró que todos los tratamientos con tocosh muestran efecto citoprotector significativo, comparadas con el control positivo. Se puede apreciar que la dosis de $1800 \mathrm{mg} / \mathrm{kg}$ de tocosh/ peso del animal tiene mayor actividad citoprotectora, seguida de la dosis de 2 $700 \mathrm{mg} / \mathrm{kg}$ y después la de $900 \mathrm{mg} / \mathrm{kg}$. La evidencia del efecto citoprotector del tocosh de papa se puede apreciar en las diferentes fotografías. En la foto 1 se muestra la mucosa sin afectación por el etanol y en la foto 2 se muestra la mucosa después de la injuria por etanol, que corresponde al control positivo y se evidencia áreas de ulceración e irritación.

En nuestro modelo experimental hemos usado etanol $70 \%$ para inducir injuria en la mucosa gástrica, por ser una sustancia que produce daño por mecanismos independientes a la acidez gástrica, sino por erosión, factor importante en la ulcerogénesis; esta característica permite evaluar el efecto citoprotector propuesto para varios grupos de fármacos usados en las gastropatías y úlcera péptica, incluyendo sucralfato, carbenoxolona y una suspensión de antiácido ${ }^{(25)}$.

Con los resultados del área de protección y lesión (tabla 2) determinamos que la dosis de $1800 \mathrm{mg} / \mathrm{kg}$ fue la que tuvo un mejor efecto citoprotector y al comparar dicho resultado con el sucralfato a dosis $30 \mathrm{mg} / \mathrm{kg}$, las áreas no

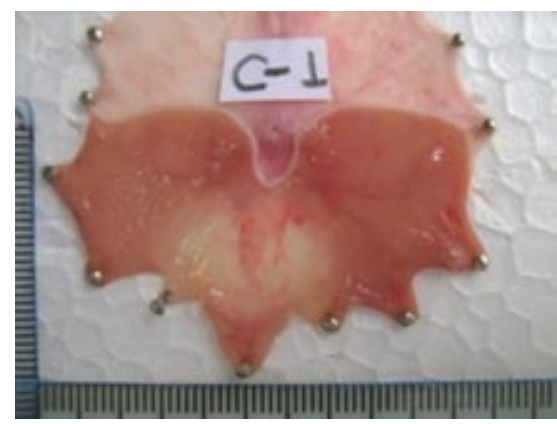

Foto 1. Tejido normal.

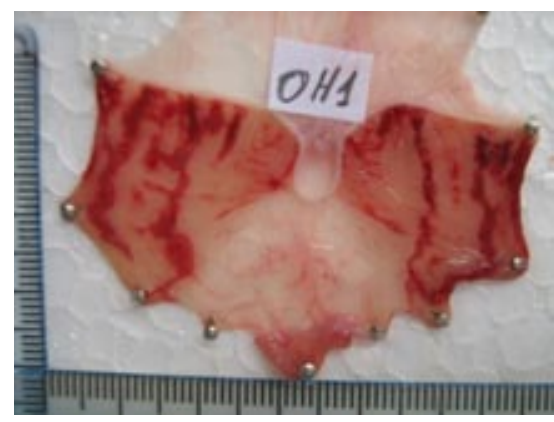

Foto 2. Tejido con lesión causada por etanol.

tuvieron diferencia significativa. Por lo que podemos afirmar que el tocosh protegió a la mucosa gástrica en la misma magnitud que el sucralfato, como se muestra en las fotos 3 y 4 .

La citoprotección que confiere el tocosh de papa la realiza mediante la formación de una capa de protección física a la mucosa, como lo hacen algunos fármacos como el sucralfato. La capacidad protectora del sucralfato contra la injuria de la mucosa gástrica por etanol ha sido comunicada previamente por Salomón y col. ${ }^{(25)}$; aunque el mecanismo no ha sido claramente establecido, se puede incluir la formación de una 'barrera protectora' en la mucosa lesionada, desactivación y unión con pepsina y ligazón con ácidos biliares ${ }^{(26)}$. Información reciente sugiere que sucralfato puede incrementar la producción de prostaglandinas E2 y mucus gástrico ${ }^{(17)}$. Estas acciones pueden explicar no solamente el efecto terapéutico sino la eficacia profiláctica del sucralfato en la prevención de la recurrencia de la úlcera.

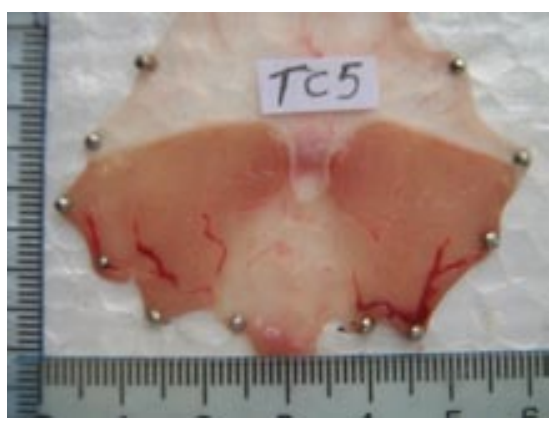

Foto 3. Tejido con protección por tocosh.

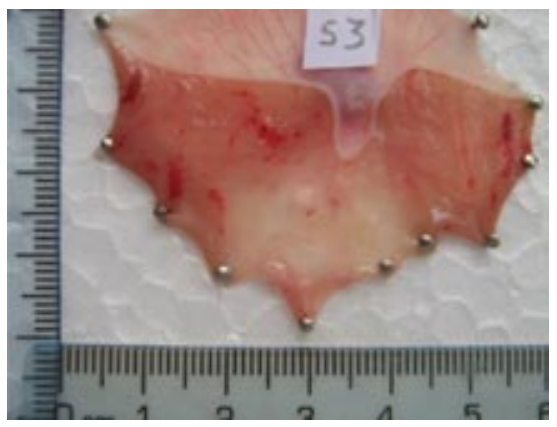

Foto 4. Tejido con protección por sucralfato.

En nuestro estudio hemos observado la adherencia del gel de tocosh en la mucosa gástrica. Es congruente atribuir la formación de esta barrera por la característica mucilagenosa referida en párrafos anteriores y esta sería la razón de la acción demulcente provocada por la amilosa y amilopectina contenidos en el tocosh, pues durante la decocción se forma una solución coloidal. Sin embargo, los mecanismos de protección podrían ser múltiples y requieren mayores estudios.

Se ha comunicado que el precipitado de zumo de papa S. tuberosum en dosis de $20 \mathrm{~mL} / \mathrm{kg}$ puede proteger hasta un $73,8 \%$ de la mucosa ${ }^{(22)}$. A diferencia de este, los resultados obtenidos con el tocosh son mejores pues evidencian una citoprotección de hasta un $97,07 \%$, por lo que podemos afirmar que la especie en sí, S. tuberosum, tiene actividad citoprotectora en la mucosa gástrica. Dicha actividad se debe principalmente a los componentes del almidón y su capacidad de formar una barrera protectora en la mucosa. 
Con respecto a las dosis administradas, Gutiérrez y col. ${ }^{(13)}$ realizaron un estudio toxicológico usando el modelo de toxicidad aguda a dosis límite de 2000 $\mathrm{mg} / \mathrm{kg}$ que, al corte anatomopatológico de hígado, pulmón, riñón y estómago, no presentó daños en tejidos y estructuras. Sobre la base de este estudio, podríamos afirmar que los tratamientos son inocuos, a pesar de haberse administrado dosis altas. Además, el tocosh en decocción (mazamorra) es consumido por la población andina de manera ancestral, por lo que descartamos una toxicidad potencial.

Finalmente, bajo nuestro modelo experimental y en las condiciones del estudio podemos concluir que el tocosh de papa Solanum tuberosum posee capacidad antioxidante y es citoprotector en la mucosa gástrica de animales de experimentación; con lo cual se estaría confirmando el conocimiento ancestral de la medicina tradicional, que el consumo de tocosh protege la mucosa gástrica en los seres humamos.

\section{REFERENCIAS BIBLIOGRÁFICAS}

1. Consejo Nacional del Ambiente, CONAM. 2001 Perú: Estrategia Nacional sobre Diversidad Biológica. Primera edición: octubre 2001. Disponible en: http://www.sernanp.gob.pe/sernanp/archivos/biblioteca/publicaciones/DOC_VARIOS/ENDB.pdf

2. Velásquez D, Casas A, Torres J, Cruz A. Ecological and socio-cultural factors influencing in situ conservation of crop diversity by traditional Andean households in Peru. J Ethnobiol Ethnomed. 2011 Dec 6;7:40. doi: 10.1186/1746-4269-7-40.

3. Garcia A, Esmérita FM, Isidro G, Edinson D. Técnicas de conservación de alimentos: El Tocosh. Lima: Universidad Nacional Federico Villarreal UNFV; 2005

4. Naupari G, Arias G, Amarillo A. Estudio químico bromatológico y de la actividad antimicrobiana de tocosh. Bol Soc Quim Perú. 1993;59(2):87-92

5. Fries AM. Compilador. De la chacra al fogón. Perú: Proyecto Andino de Tecnologias Campesinas PRATEC; 2001.

6. Manrique I, Rosales P. Evaluación tecnológica de tocosh. Lima: Fundación para el desarrollo del agro FUNDEAGRO. Resumen de investigaciones apoyadas por FUNDEAGRO. 1993:149-50.

7. Mori P, Malena M. Estudio del efecto de Tocosh de papa como probiótico en el control del peso corporal y mayor crecimiento en ratas jóvenes frente a cultivo de Lactobacillus acidophillus. En: V Congreso Mundial de Medicina Tradicional. Lima: Facultad de Medicina Humana, Universidad San Martín de Porres; 2005:24, 25.

8. Valdivia M. Gastritis y gastropatias. Rev Gastroenterol Perú. 2011;31 (1):38-48

9. Carhuapoma M. Composición química, actividad anti-Helicobacter pylori del aceite esencial de Satureja brevicalyx Epling "urqu muña" (tesis doctoral). Lima: Facultad de Farmacia y Bioquímica, Universidad Nacional Mayor de San Marcos; 2007.

10. Fuentes E, Camorlinga M, Maldonado C. Infección, inflamación y cáncer gástrico. Salud pública Méx (Revista en internet) ; 2009 Octubre (acceso 03 de noviembre de 2013) ;51(5):427-33. Disponible en: http://dx.doi.org/10.1590/S003636342009000500010

11. Lock O. Investigación Fitoquímica: Método en el estudio de productos naturales. Lima: Segunda edición. Editorial Fondo. Pontificia Universidad Católica del Perú; 1994.

12. Sigh J, Kaur L. Compiladores. Advances in potato chemistry and technology. 1ra edición. United States of America: Elsevier Inc; 2009.

13. Gutiérrez D. Análisis toxicológico y estudio fitoquimico del extracto hidroalcohólico de Tocosh. Perú: Universidad Wiener; 2010.

14. Rozowski J, Cuevas A, Castillo O, Marin P, Strobel P, Pérez D y col. Diferencias en antioxidantes plasmáticos según nivel socioeconómico en mujeres chilenas. Rev méd Chile (revista en internet). 2001 Enero (acceso 18 de noviembre de 2013); 129(1):43-50. Disponible en: http://dx.doi. org/10.4067/S0034-98872001000100006.

15. Gutierrez C, Raynal A. Gastroprotective effect of intragastric clarithromtycin against damage induced by etanol in rats. Digestive Diseases and Sciences. 1999;44(8):1721-31.

16. Hernandez R, Montiel C, Vásquez O. Gastric mucosal cell proliferation in etanol-induced chronic mucosal injury is related to oxidative stress and lipid peroxidation in rats. Labs invest. 2000 Aug;80(8):1161-9. Citado en PubMed PMID:10950107.

17. Brown C. Antioxidants in potato. Am J Potato Res. 2005;82:163-72.

18. Navarre D, Goyer A, Shakya R. Nutritional value of potatoes: vitamin, phytonutrient, and mineral content. En: Sigh J, Kaur L. Compiladores. Advances in potato chemistry and technology. 1 ra edición. United States of America: Elsevier Inc; 2009:395-424.

19. Coetzer C, Corsini D, Love S, Pavek J, Tumer N. Control of enzymatic browning in potato (Solanum tuberosum L.) by sense and antisense RNA from tomato polyphenol oxidase. J Agric Food Chem. 2001;49(2):652-7.
20. Andre C, Oufir M, Guignard C, Hoffman L, Hausman J, Evers D, et al. Antioxidant profiling of native Andean potato tubers (Solanum tuberosum L.) reveals cultivars with high levels of $\beta$-carotene, $\alpha$-tocopherol, chlorogenic acid, and petanin. J Agric Food Chem. 2007;55:10839-49.

21. Senol A, Isler M, Karahan A, Kilic G, Kuleasan $H$, Kaya S, et al. Preventive effect of probiotics and $\alpha$-tocopherol on ethanol-induced gastric mucosal injury in rats. J Medicinal Food. 2011 Jan;14(1/2):173-9. Citado en PubMed PMID: 21244242

22. Sandoval M, Huamán $\mathrm{O}$, Oré R, Loli A, Ayala S. Efecto antioxidante y citoprotector del Solanum tuberosum (papa) en la mucosa gástrica de animales de experimentación. An Fac med. 2010;71(3):147-52.

23. Rosenthal S, Jansky S. Effect of production site and storage on antioxidant levels in specialty potato (Solanum tuberosum L.) tubers. Sci Food Agric. 2008;88:2087-92

24. Sandoval M, Ayala S, Oré R, Loli A, Huamán O, Valdivieso $R$ y col. Capacidad antioxidante de la sangre de grado (Croton palanostigma) sobre la mucosa gástrica, en animales de experimentación. An Fac med. 2006;67(3):199-205.

25. Ayala S, Díaz D, Palomino M, Armas S, Paz J. Efecto protector de Croton palanostigma y Aloe vera frente a injuria aguda de mucosa gástrica inducida por etanol en ratas. An Fac med. 1999; $60(1): 22-9$.

26. Quillama E, Dávila S, Medina A, Ávalos C, Paredes D. Evaluación de la biodiversidad láctica de "tocosh", alimento fermentado tradicional de Perú. En: resúmenes Biotecnologia: XXI Reunión científica del Instituto de Investigación de ciencias Biológicas Antonio Raimondi. Lima; Universidad Nacional Mayor de San Marcos; 2012. p. 80.

Seleccionado como el mejor trabajo de investigación en Ciencias Básicas, XIII Jornadas Cientificas Sanfernandinas, SVI Jornadas de Investigación en Salud, XXIII Jornadas Sanfernandinas Estudiantiles 'Dr. Abelardo Tejada Valencia', Facultad de Medicina, Universidad Nacional Mayor de San Marcos, septiembre de 2014.

Conflictos de interés: Los autores declaran no tener conflictos de interés.

\section{Correspondencia:}

Miguel Hernán Sandoval Vegas.

Celular: 998731434

Teléfono UNMSM: 6197000 - 4684.

Correo electrónico:mhsave@gmail.com;msandovalv@ unmsm.edu.pe 